\title{
Incidencia de la gastronomía para elegir un destino. Caso de estudio: Baños de Agua Santa. Tungurahua-Ecuador
}

Incidence of gastronomy to choose a destination. Case study: Baños de Agua Santa. Tungurahua-Ecuador

Jessica Alexandra Robalino Vallejo. ${ }^{1}$, César Felipe Rivadeneira Yánez. ${ }^{2}$, Ana Matilde Moreno Guerra. ${ }^{3}$ \& Andrea Estefanía Fierro Ricaurte. ${ }^{4}$

\section{Abstract. $\quad$ DOI: $\underline{\text { https://doi.org/10.33262/concienciadigital.v3i2.1.1217 }}$}

The starting point for this work on the nature of the demand for the product of tourist gastronomy is the motivation that tourists have for destinations and experience their gastronomy. Based on this argument, the investigation is carried out in the City of Baños de Agua Santa, canton of the Province of Tungurahua, considered one of the most visited tourist destinations in Ecuador. The research is carried out considering a sample of tourists to whom a survey is applied to determine the motivations of their trip. In the same way, we proceed with the legally registered restaurants to which a similar instrument is applied in order to know the gastronomic offer. The results obtained indicate the relevant aspects of the situation of gastronomy in the city of Baños de Agua Santa, establishing that it is an important element in the environment and development of tourist activities to support the operation, it presents a variety of national and international meals seeking to meet the requirements of visitors. When comparing the results obtained and performing a contrast analysis between the type of tourists and the type of gastronomy, it is established that, although gastronomy is an important element that supports the tourist activity of the city, it is not a determining factor as for tourists to choose the destination for this reason. However, it is concluded that the gastronomy managed in an adequate way, maintaining the quality levels of the offer of dishes

\footnotetext{
${ }^{1}$ Escuela Superior Politécnica de Chimborazo, Facultad de Salud Pública, Carrera Gastronomía, Riobamba, Ecuador, jrobalino@espoch.edu.ec

2 Investigador independiente, Riobamba, Ecuador, chef@feliperivadeneira.com

${ }^{3}$ Escuela Superior Politécnica de Chimborazo, Facultad de Salud Pública, Carrera Gastronomía, Riobamba, Ecuador, amoreno_g@espoch.edu.ec

${ }^{4}$ Investigador independiente, Riobamba, Ecuador, andreafierro18@hotmail.com
} 
and the quality of service is an important promotional element that, due to its characteristics of sensory experience, is able to enhance the destination.

Keywords: Gastronomy, Tourist Destination, Travel Motivations, Tourist

\section{Resumen.}

El punto de partida para este trabajo sobre la naturaleza de la demanda del producto de gastronomía turística es la motivación que los turistas tienen para los destinos y experimentar su gastronomía. Con base a este argumento se realiza la investigación en la Ciudad de Baños de Agua Santa, cantón de la Provincia de Tungurahua considerado como uno de los destinos turísticos más visitados en el Ecuador. La investigación se realiza considerando una muestra de turistas a los que se les aplica una encuesta para determinar las motivaciones de su viaje. De igual manera se procede con los restaurantes legalmente registrados a los que se les aplica un instrumento similar con el propósito de conocer la oferta gastronómica. Los resultados obtenidos indican los aspectos relevantes de la situación de la gastronomía en la ciudad de Baños de Agua Santa estableciéndose que es un elemento importante en el entorno y desarrollo de las actividades turísticas como soporte de la operación, presenta una diversidad de comidas nacionales e internacionales buscando satisfacer los requerimientos de los visitantes. Al comparar los resultados obtenidos y realizar un análisis de contraste entre el tipo de turistas y el tipo de gastronomía se establece que, sin embargo de ser la gastronomía un elemento importante que da sostenimiento a la actividad turística de la ciudad, no es un factor determinante como para que los turistas elijan el destino por esta razón. Sin embargo se concluye que la gastronomía manejada de forma adecuada, manteniendo los niveles de calidad de la oferta de platos y la calidad de servicio se constituye en un elemento promocional importante que por sus características de experiencia sensorial es capaz de potenciar el destino.

Palabras claves: Gastronomía, Destino Turístico, Motivaciones del viaje, Turista.

\section{Introducción.}

La literatura turística ha documentado ampliamente que las especialidades locales de comida y gastronomía inciden significativamente en la elección del destino de vacaciones (Norrid, 2017; Bailón \& Rojas, 2017; Fusté F, 2017; Krauser R \& Bahls A, 2016) he influyen enormemente en el tiempo y en el disfrute de la estancia (Arango R, Africano G, \& Viana A, 2018). También se enfatiza ampliamente sobre la importancia de los productos locales y la gastronomía como fuerza impulsora del crecimiento económico. en los centros de turismo comunitario por ejemplo, las actividades agrícolas ayudan a preservar el equilibrio ambiental y la conservación del suelo, que son requisitos previos y necesarios para el desarrollo del turismo. Dicho esto, la agricultura, la industria alimentaria y el turismo son, por lo tanto, complementarios, y se caracterizan por vínculos ascendentes y descendentes y fuertes efectos multiplicadores intersectoriales (Medina F, 2017). 
En muchos destinos turísticos, el desarrollo y la promoción de productos locales han crecido en paralelo con el reconocimiento y la importancia de la gastronomía para fidelizar al cliente y atraer a nuevos visitantes (Juárez L \& Cebrián R, 2016). En el lugar de vacaciones que el turista elige, las especialidades culinarias locales ayudan a transmitir una sensación de autenticidad y singularidad, y refuerzan la imagen externa del territorio (Uribe A, Avalos E, \& Villa J, 2017).

Más allá de lo estrictamente turístico en los destinos, la gastronomía se ha convertido en un canal promocional adicional, debido al importante papel que desempeñan los productos alimenticios locales en las economías turísticas locales, en los últimos años se constituyen cada vez más en herramientas de marketing y marca para la promoción del turismo (Sobrado D, 2018; Díaz M \& García J, 2015; Mascarenhas R \& Gândara J, 2015).

Dado que las especialidades gastronómicas locales evocan la identidad, la cultura y la sostenibilidad del lugar de origen, muchos autores sugieren que deben considerarse explícita e implícitamente cuando se diseñan las estrategias de comercialización de los destinos locales y regionales. Además, las denominaciones de autenticidad y las etiquetas de calidad son capaces de promover con frecuencia el turismo y transmitir un sentimiento de calidad y tipicidad (Vázquez M, 2017)

Contrariamente a la cocina local que no es realmente comercializable fuera de los pequeños restaurantes de una región turística, las especialidades locales de gastronomía pueden comercializarse fuera del lugar de origen. La disponibilidad de estos productos en las tiendas de alimentos cerca de sus hogares permite a los posibles visitantes tener una idea de las tradiciones locales a las que representan y, por lo tanto, aumentar su percepción de la autenticidad y el atractivo del supuesto lugar de origen. Además, para aquellos viajeros que ya han pasado unas vacaciones en un destino turístico, el consumo de los productos locales del sitio que visitaron puede ayudar a mantener vivo el interés en ese destino. En ambos casos, estos productos alimenticios locales deberían hacer que sea más probable que los clientes visiten (o vuelvan a visitar) un lugar turístico (Norrid, 2017).

Aunque hay muchos trabajos de investigación que destacan los beneficios de la comida local en el turismo, a través del análisis e implementación de rutas gastronómicas o el estudio de la gastronomía típica vinculada a la preservación de los recursos ancestrales, la cultura y el turismo, se ha dedicado muy poca atención a verificar la existencia de un efecto estadísticamente significativo de los productos gastronómicos locales en la intención de elegir o visitar un destino turístico (Franco C , Francio C, \& Torres N, 2017; Kido C, Díaz C, \& Kido C, Una Nueva Interpretación de La satisfacción del comensal como elemento clave del binomio gastronomíaturismo en Tijuana., 2019). Los estudios realizados encuentran una correlación positiva entre la percepción de la calidad de los alimentos y la intención de volver a visitar. Por otro lado la Literatura existente aún no ha analizado qué efecto tienen los productos locales en los destinos turísticos competitivos. 


\section{La Gastronomía una experiencia turística sensorial}

A medida que aumenta la competencia entre los destinos turísticos, la cultura local se está convirtiendo en una fuente cada vez más valiosa de nuevos productos y actividades para atraer y divertir a los turistas. La gastronomía tiene un papel particularmente importante que desempeñar en esto, no solo porque la comida es fundamental para la experiencia turística, sino también porque la gastronomía se ha convertido en una fuente importante de formación de identidad en las sociedades posmodernas.

En la actualidad se habla de que el ser humano es lo que come no solo en el sentido físico, sino también porque se identifica con ciertos tipos de cocina que encuentra en las visitas a los destinos turísticos (Serra J \& Pascual M, s/f). A medida que los turistas se vuelven más móviles, también lo hacen los alimentos que comen. La asociación de ciertos alimentos con regiones particulares está siendo desafiada por la creciente movilidad de los alimentos, los estilos culinarios y la progresiva diferenciación de platos y cocinas. Lejos de producir un paisaje gastronómico homogeneizado, la tensión entre globalización y localización está produciendo cada vez más variaciones (Norrid, 2017).

No solo están surgiendo bebidas y alimentos globales, como Coca-Cola y McDonald's, sino que la comida local y regional está prosperando, y también se están creando nuevos alimentos de fusión para satisfacer las necesidades del "alma global" (Iyer 2000). Los turistas mismos están contribuyendo a la movilidad gastronómica, al crear una demanda en sus propios países de alimentos que han encontrado en el extranjero (Ricon, Santos, \& Galinmdo, 2016).

Por otro lado la comida estructura el día turístico. Una gran parte de las experiencias turísticas se gastan en consumir alimentos y bebidas, o decidir qué y dónde consumir. Sin embargo, muchas de estas experiencias se dan por sentadas, porque a menudo el turista considera que comer es una necesidad más que una actividad de ocio. Una de las tareas esenciales en el desarrollo y comercialización del turismo gastronómico, por lo tanto, es encontrar formas de agregar valor a la experiencia gastronómica para que sea memorable (Kido C, Carrión I, \& Kido C, La satisfacción del comensal como elemento clave del binomio gastronomía-turismo en Tijuana., 2018).

Si el turismo gastronómico pasa de la prestación de servicios a la puesta en escena de experiencias, la calidad de los elementos básicos del producto se dará cada vez más por entendidas para los consumidores, que exigirán experiencias intensamente atractivas como parte del producto turístico y gastronómico (Fusté F, 2017). El desarrollo de experiencias gastronómicas se hace evidente en la aparición de restaurantes que ofrecen un paquete total de comida, entretenimiento y ambiente. Crear experiencias vendibles también se convierte en parte del arte de desarrollar destinos turísticos. A medida que aumenta la competencia global entre los destinos turísticos, la búsqueda de productos distintivos se vuelve más intensa. La gastronomía es vista como una fuente importante de imágenes y experiencias comercializables para los turistas (Sobrado D, 2018) 
Las experiencias se pueden desarrollar mediante la vinculación de recursos en una sola región o en diferentes regiones a través de rutas gastronómicos. El turista puede experimentar una gama de productos gastronómicos vinculados a los recursos culturales y medio ambientales de una región específica, como es el caso de Baños de Agua Santa, o comparar la forma en que la cultura interactúa con productos gastronómicos similares en diferentes regiones. Crear tales experiencias requiere no solo vincular diferentes territorios sino también vincular a diferentes productores en la cadena de valor (Varisco, 2015).

Las experiencias gastronómicas para los turistas generalmente se desarrollan desde la perspectiva de aspectos "únicos" de la culinaria que solo se pueden encontrar en ese lugar en particular. Sin embargo, a medida que más destinos desarrollan experiencias gastronómicas para los visitantes, el problema de la propiedad intelectual se agudiza y la cocina regional y local se internacionaliza.

\section{La gastronomía utilizada para vender el destino}

Si la gastronomía se puede vincular a países o regiones específicas, se convierte en una poderosa herramienta de marketing turístico. La autenticidad siempre se ha visto como un aspecto importante del consumo turístico, y buscar alimentos locales y regionales "auténticos" puede convertirse en un motivo para visitar un destino en particular. Muchos países y regiones de todo el mundo han comenzado a darse cuenta de esto, y están utilizando la gastronomía para promocionarse (Curiel, 2015)

Gran parte del esfuerzo de marketing dirigido al desarrollo de la gastronomía en poblaciones pequeñas tiene como objetivo establecer y aumentar la "autenticidad" de la cocina local. Un aspecto importante del desarrollo de productos locales auténticos es un atractivo para la nostalgia y las virtudes de un pasado vivencial tradicional, más simple y más saludable.

En la actualidad una gran cantidad de destinos utilizan la gastronomía como una herramienta de marketing turístico, y muchos también utilizan el turismo para promover la gastronomía. Sin embargo, hay indicios de que tales esfuerzos de marketing son mucho menos efectivos de lo que podrían ser. Debido a la separación de los campos del turismo y la gastronomía, los comercializadores de turismo generalmente no entienden el producto gastronómico, y muchos gastrónomos no entienden el turismo (Sobrado D, 2018).

\section{La situación de la Gastronomía en Baños de Agua Santa}

Rankeada entre los 10 primero destinos turísticos del Ecuador (Enviajes, 2020), la ciudad de Baños de Agua Santa es un rincón paradisiaco que une la región sierra con la amazonia, se encuentra a $180 \mathrm{Km}$ de la capital del Ecuador Quito y a $35 \mathrm{Km}$ de la ciudad de Ambato, capital de la provincia de Tungurahua, con una población aproximada de 20.000 habitantes que se dedican en un $90 \%$ a la actividad turística, haciendo de esta ciudad un emporio para los emprendedores turísticos. 
Famosa por las aguas termales producto de su cercanía al volcán Tungurahua, sus artesanías y los “Alfeñiques” Arango R, 2019 la ciudad de Baños inicia su desarrollo turístico en la década del 70 del siglo XX, siendo un punto estratégico de ingreso a la amazonia ecuatoriana, concentra a viajeros de paso y turistas nacionales, especialmente de la zona sierra centro, por sus características climáticas se constituye en el espacio geográfico más adecuado para que los turistas extranjeros se aclimaten antes de viajar a la amazonia, estos dos aspectos coyunturales hicieron posible que a finales del siglo XX la ciudad de Baños se haya consolidado como un de los destinos turísticos más importantes del Ecuador.

A inicios del año 2000, la crisis de desplazamiento provocada por la erupción del volcán Tungurahua, obliga a los habitantes a abandonar el poblado, frenándose el desarrollo turístico por varios meses, sin embargo los habitantes, rompiendo los protocolos de seguridad retornan a la ciudad que paulatinamente fue recuperando su ritmo habitual, con la salvedad de tener un atractivo turístico adicional, el proceso eruptivo del volcán Tungurahua, que ha contribuido en las dos últimas décadas a la construcción de una ciudad pequeña en extensión pero cosmopolita en su cotidianidad, es un espacio urbano que hace posible la operación de una gran cantidad de actividades turísticas, sobre todo vinculadas a la naturaleza y a los deportes extremos.

Al configurarse la Ciudad de Baños de Agua Santa con la influencia de los visitantes nacionales de las tres regiones y visitantes extranjeros provenientes de distintos puntos del planeta, la gastronomía de la ciudad toma matices diversos e inverosímiles, en lo que respecta a la cocina típica recibe la influencia de la región Sierra Centro sobre todo de la ciudad de Ambato, se puede encontrar gastronomía de la región amazónica, o platos típicos del Austro o la Región Litoral. La afluencia cada vez mayor de turistas extranjeros a dado lugar a la apertura de restaurantes especializados en comida internacional, que en muchos de los casos están administrados por extranjeros que vieron una oportunidad de negocios y decidieron quedarse en la ciudad y hacer propuestas gastronómicas interesantes, o restaurantes con oferta gastronómica mixta, es así que en baños se puede encontrar desde el simple de los platos típicos nacionales al más complejo potaje japones.

De acuerdo al Catastro del Ministerio de Turístico de la Ciudad de Baños de Aguas Santa (MINTUR, 2018), existen 180 restaurantes de los cuales el 77\% pertenecen a la tercera categoría, $12,54 \%$ a la segunda Categoría y $10,46 \%$ a la cuarta categoría, no se reportan restaurantes de primera categoría. En lo que se refiere a las especialidades que se sirven en estos locales, no existe una referencia específica que permita determinar este parámetro, lo que si se reporta es que una gran mayoría de estos locales oferta comida típica y al mismo tiempo comida internacional, considerando que deben ajustarse a la variedad de la demanda (Povea T , 2018).

En la búsqueda de un plato típico que represente a la ciudad de Baños de Agua Santa, la literatura reporta que están arraigados algunas preparaciones que forman parte del Menú característico de la Región Sierra Centro del Ecuador, Para citar algunos ejemplos interesantes, se encuentran el cuy 
con papas, la fritada de cerdo, el hormado de cerdo, las papas con cuero entre otros (Hurtado V \& Molina C, 2018; Povea T , 2018; Masabanda T, 2015; Rivera P, 2013; Silva S, 2016).

Uno de los aspectos que caracteriza a la Ciudad de Baños de Agua Santa, son los típicos alfeñiques o melcochas, es un preparado de azúcar de caña, al que se le carameliza y se lo va moldeando hasta que alcanza un punto de cristalización, a este producto se le añaden especias fragantes para que agarre un sabor especial, generalmente mandarina, esta preparación, se hace en otras ciudades e inclusive en otros países, sin embargo y sobre todo para el turista nacional, su consumo es una tradición arraigada.

Arango R, 2019 el Alfeñico o Melcocha es una especie de caramelo o confitura con base en azúcar pura de la caña preparada en pasta alargada y retorcida a la que pueden sumarse otros componentes; es una confitura folclórica desde tiempos coloniales.

\section{Metodología}

El proceso metodológico empleado en esta investigación, está vinculado a las motivaciones por las cuales el turista decide dirigirse a un destino en específico, y si en esa decisión uno de los factores que influye es la gastronomía, en este contexto la investigación requirió de información de primera mano de los turistas y la oferta de restauración de la ciudad de Baños de Agua Santa. El trabajo se realizó de junio a noviembre del 2019.

La población de los turistas se consideró en función de la demanda real diaria de plazas en los restaurantes de la ciudad de Baños de Agua Santa que es de 6.211 sillas, 1.570 mesas en 180 locales dando una muestra de 71 turistas hombres y mujeres de entre 16 a los 75 años. Se extrajo una muestra por conveniencia de los 180 restaurantes de la ciudad de Baños considerando las características de oferta, en función del tipo de comida que se sirve en ellos,

Internacional, ecuatoriana, de autor y mixta dando un total de 56 establecimientos ubicados en los sectores con mayor afluencia turística. Los datos para el muestreo fueron obtenidos del catastro de servicios turísticos del MINTUR (2018).

Para obtener la información pertinente se estructuraron instrumentos de recolección de datos, en los dos casos se utilizó la técnica de la encuesta con cuestionarios dirigidos a los turistas y a los servidores de restauración, cada cuestionario cuento con preguntas de respuestas simples y fue estructurado de tal forma que el análisis del cuestionario sea rápido y de fácil contestación, los cuestionarios se realizaron en español e inglés, Los cuestionarios fueron validados con la aplicación del 5\% de la muestra, para evitar errores de adquisición de datos y de dificultades en el tratamiento de la información.

Para dar complementariedad a la información se utilizó alternativamente información estadística del MINTUR y de otros trabajos de investigación que posibilitaron ampliar el análisis y dar fluidez 
al proceso de interpretación de la situación actual de la gastronomía en la ciudad de Baños de Agua Santa.

En el tratamiento de la información se utilizaron recursos informáticos apropiados para la estructuración, análisis e interpretación de la base de datos obtenida, se manejaron estadísticos descriptivos y análisis univariante y bivariantes a través de la aplicación de una prueba no paramétrica de contraste chi cuadrado y análisis correlacional, que permitieron la comprobación de la hipótesis propuesta "La Gastronomía incide en la elección del destino turístico.

\section{Resultados}

\section{Análisis de la Oferta}

Figura 1 Planta Turística de la Ciudad de Baños de Agua Santa

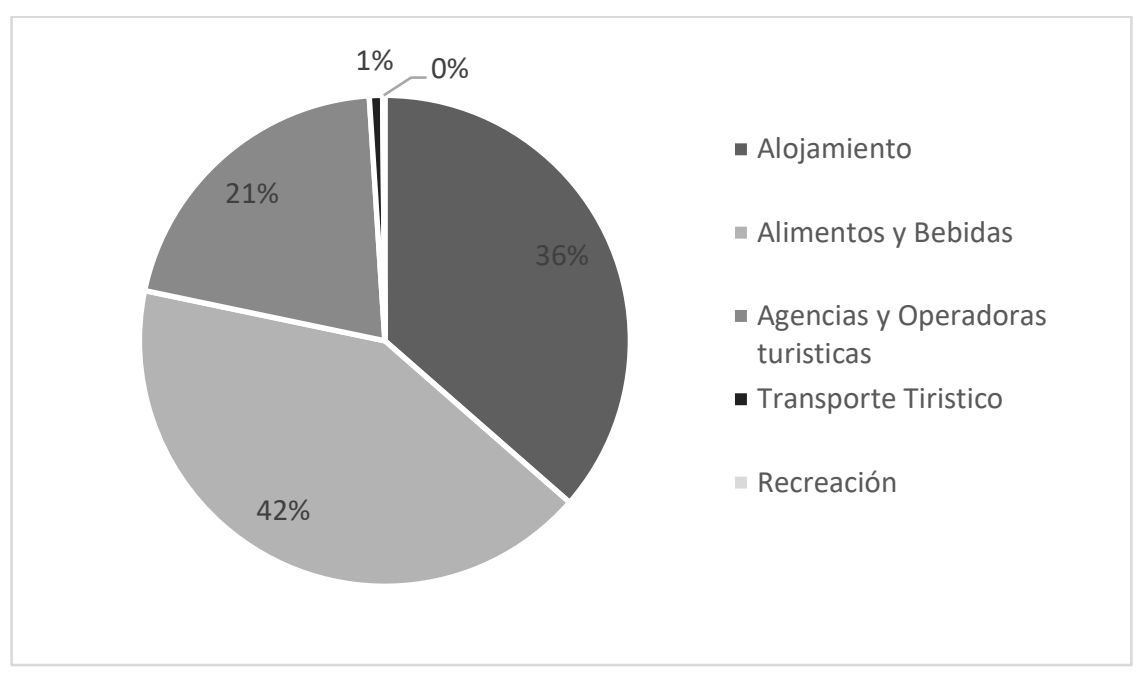

Fuente: Mintur (2018)

De acuerdo a los datos del catastro de servidores turísticos del MINTUR (2018), La ciudad de Baños de Agua Santa cuenta con una planta turística importante, en relación al número de habitantes por lo que es, a simple vista, una población con vocación eminentemente turística, Se reporta 686 servidores turísticos legalmente registrados de los cuales el $42 \%$ pertenece los servidores de alimentos y bebidas, el $36 \%$ a alojamiento, el $21 \%$ a agencias y operadoras el $1 \%$ transporte Turístico y 0,15\% distracción, como se puede observar en la Figura 1.

Los servidores turísticos orientados al servicio de la alimentación y bebidas son 180 restaurantes, bares, cafeterías, discotecas y fuentes de soda, que han sido debidamente categorizados, por los organismos reguladores competentes ubicándolos en la segunda, tercera y cuarta categoría, el número de plazas en mesas y sillas de cada uno de estos rubros se propone en la Tabla 1. 
Vol. 3, $\mathrm{N}^{\circ}$ 2.1, p. 6-24, mayo, 2020

Tabla 1 Catastro de las categorías de establecimientos de comidas y bebidas en el cantón Baños

\begin{tabular}{llll}
\hline Clase de Establecimiento & Cantidad & $\mathbf{N}^{\circ}$ de Mesas & $\mathbf{N}^{\circ}$ de Plazas \\
\hline Restaurante & 180 & 1.570 & 6.211 \\
Bar & 37 & 241 & 964 \\
Cafetería & 26 & 170 & 662 \\
Discoteca & 22 & 308 & 1.232 \\
Fuente de Soda & 22 & 99 & 396 \\
TOTAL & 287 & 2.388 & 9.465 \\
\hline
\end{tabular}

Fuente: MINTUR (2018)

En la tabla 1 se reportan un total de 180 restaurantes con 1.570 mesas y 6.211 plazas (sillas) que indican la importante capacidad operativa que tiene la oferta de restauración formal en la ciudad de Baños de Agua Santa, que en relación a la cantidad de población es proporcionalmente mayor que al que tienen las ciudades de Ambato y Riobamba (MINTUR, 2018). Esta capacidad instalada refleja la importancia de la gastronomía en el entorno en el que se desarrollan las diferentes actividades turísticas de la ciudad de Baños, sin embargo es un factor complementario para el turismo y no determinante en la decisión de los turistas para elegir este destino, a continuación se presentan algunos factores de análisis relacionados con la oferta turística que así lo demuestran.

Tabla 2 Oferta gastronómica en la ciudad de Baños de Agua Santa

\begin{tabular}{lll}
\hline Tipo de oferta & Numero & Porcentaje \\
\hline Internacional & 23 & 41,07 \\
Ecuatoriana & 16 & 28,57 \\
Mixta & 10 & 17,85 \\
De Autor & 7 & 12,5 \\
Total & 56 & 100 \\
\hline
\end{tabular}

Fuente: Encuesta a la oferta gastronómica 
La tabla 2 indica que la oferta gastronómica que predomina es la internacional, ya que Baños de Agua Santa es considerada un destino importante para los turistas internacionales. Los mismos que por general prefieren comida que ellos conocen (por seguridad, por tradición, por desconocimiento, etc.), por lo que ha llevado a la apertura de estos tipos de restaurantes como oferta prioritaria. Es importante hacer notar que una buena parte de estos restaurantes de comida internacionales ofertan comida rápida, italiana y norteamericana que esta dirigida a consumidores jóvenes, que representan un importante segmento de la demanda de turismo nacional e internacional del cantón Baños (Masabanda T, 2015).

Tabla 3 Rango de precios en la oferta gastronómica

\begin{tabular}{lll}
\hline Precio & Numero & Porcentaje \\
Bajo & 16 & 28,57 \\
Medio & 37 & 66,07 \\
Alto & 3 & 0,53 \\
Total & 56 & 100
\end{tabular}

Fuente: Encuesta a la oferta gastronómica

El rango de precio con mayor oferta fue el medio que implica precios que van de $\$ 4,00$ a $\$ 12,00$ dólares de consumo promedio. La generalización de este rango de precios en la oferta gastronómica tiene varios factores que pueden ser analizados, el primero y más evidente es que los clientes prefieren al momento de escoger un restaurante, un casual e informal, también este rango de precios permite mantenerse a los servidores frente a la competencia. Si se evalúa este indicador con objetividad, dice mucho sobre el tipo de turistas que forman parte de la demanda gastronómica que generalmente son jóvenes, que limitan sus presupuestos para alimentación destinándolos a actividades que están más vinculadas a los deportes de aventura, por otro lado los costos propuestos por la oferta de restauración permiten una variedad de platos limitados que generalmente están asociados al consumo de almuerzos, ciertos platos típicos y comida rápida. El análisis de estos factores lleva a determinar que los costos impuestos por la oferta gastronómica están en relación a los presupuestos que los turistas están dispuestos a gastar en alimentación, en este sentido se reafirma el criterio de que la gastronomía de la ciudad de Baños de Agua Santa no es un factor determinante para la elección del destino.

Se ha determinado también el flujo de turistas en los 56 locales con un promedio diario de 30 comensales que alcanzan a 1.680 de los cuales se ha tomando una muestra de 48 turistas entre nacionales y extranjeros obteniéndose la siguiente tabla de contingencia 
Tabla 4 Tabla de contingencia Tipo de Cocina * Tipo de Turista

\begin{tabular}{|c|c|c|c|c|c|c|}
\hline \multirow{4}{*}{$\begin{array}{l}\text { Tipo } \\
\text { Cocina }\end{array}$} & \multicolumn{4}{|c|}{ de Tipo de Turista } & \multicolumn{2}{|c|}{ Total } \\
\hline & \multirow{2}{*}{\multicolumn{2}{|c|}{$\begin{array}{l}\text { Turistas } \\
\text { Nacionales }\end{array}$}} & \multirow{2}{*}{\multicolumn{2}{|c|}{$\begin{array}{l}\text { Turistas } \\
\text { Extranjeros }\end{array}$}} & \multirow[b]{3}{*}{$\mathrm{N}$} & \multirow[b]{3}{*}{$\%$} \\
\hline & & & & & & \\
\hline & $\mathrm{N}$ & $\%$ & $\mathrm{~N}$ & $\%$ & & \\
\hline Cocina & 8 & 16,7 & 13 & 27,1 & 21 & 43,8 \\
\hline \multicolumn{7}{|c|}{ Internacional } \\
\hline \multicolumn{6}{|c|}{ Nacional } & 35,4 \\
\hline $\begin{array}{l}\text { Cocina } \\
\text { Mixta }\end{array}$ & 5 & 10,4 & 4 & 8,3 & 9 & 18,8 \\
\hline $\begin{array}{l}\text { Cocina } \\
\text { Autor }\end{array}$ & de 1 & 2,1 & 0 & 0,0 & 1 & 2,1 \\
\hline Total & 23 & 47,9 & 25 & 52,1 & 48 & 100,0 \\
\hline
\end{tabular}

Fuente: Encuesta a la oferta gastronómica

En la tabla de contingencia 4 se establecen las relaciones entre el tipo de turistas nacionales e internacionales con el tipo de gastronomía que prefieren encontrándose que la relación porcentual más alta es de $27,1 \%$ de turistas extranjeros con la cocina internacional, la segunda relación importantes se da entre los turistas nacionales y la cocina nacional con 18,8\%; así mismo una relación reciproca entre la comida nacional y los turistas extranjeros $16,7 \%$ y los turistas extranjeros con la comida Nacional 16,7\%. Al aplicar el estadístico de Chi cuadrado con 3 grados de libertad se obtiene un $\mathrm{P}$ valor $=0,516$, lo que indica que entre los tipos de turistas y el tipo de gastronomía no existe una relación significativa, esto significa que la gastronomía de la ciudad de Baños de Agua Santa no es un factor determínate para elegir este destino.

\section{Análisis de la Demanda}

Considerando la muestra de turistas calculada en función de la oferta real de plazas es decir de la capacidad operativa de los restaurantes de la ciudad de Baños de Agua Santa se presentan a continuación el análisis de algunos factores que permiten establecer si existe la determinación de viaje de los turistas a este destino motivados por la gastronomía. 
Vol. 3, $\mathrm{N}^{\circ}$ 2.1, p. 6-24, mayo, 2020

Tabla 5 Motivación para llegar al destino de Baños de Agua Santa

\begin{tabular}{lll}
\hline Opción & Frecuencia & Porcentaje \\
\hline Gastronomía & 5 & 7,04 \\
Deportes Extremos & 37 & 52,11 \\
Atractivos Turísticos & 8 & 11,27 \\
Descanso & 12 & 16,90 \\
Salud & 9 & 12,68 \\
Total & 71 & 100,0
\end{tabular}

Fuente: Encuesta a la demanda gastronómica

Como lo demuestra la tabla 5, se reportan 5 motivaciones principales por los turistas nacionales y extranjeros para elegir el destino de la ciudad de Baños de Agua Santa, en primer lugar están los deportes extremos y de aventura con el 52,11\% de preferencia, seguido de el descanso con 16,90\%, turismo de salud el $12,68 \%$, los atractivos turísticos con $11,27 \%$ y en el quinto lugar la gastronomía del lugar con $7,04 \%$.

Lo que significa que la principal motivación para elegir este destino es la realización de deportes extremos, que en realidad es en donde se ha enfocado la promoción turística, es en torno a estas actividades que se han desarrollado los otros emprendimientos turísticos, entonces se toma a la gastronomía como parte de la cadena productiva del turismo en la ciudad. Esto no quiere decir que la calidad y la presentación en la prestación de los servicios de restauración sean irrelevantes, más bien es importante ir construyendo una imagen complementaria que fortalezca las actividades principales, trabajando en un beneficio mutuo.

Tabla 6 Motivos para elegir un restaurante

\begin{tabular}{lll}
\hline Opción & Frecuencia & Porcentaje \\
\hline Variedad de Platos & 60 & 38,2 \\
Precios & 34 & 21,7 \\
Ambientación & 32 & 20,4 \\
Ubicación & 31 & 19,7 \\
Total & 157 & 100,0
\end{tabular}

Fuente: Encuesta a la demanda gastronómica 
En la Tabla 6 se propone la opinión sobra la motivación que tiene el turista para tomar la decisión de ir a uno u otro local gastronómico, depende de varios factores, que considera importantes, los turistas encuestados en la ciudad de Baños de Agua Santa responden esta pregunta de opción múltiple mencionando los siguientes aspectos para elegir un restaurante.

En primer lugar proponen la variedad de platos con el 38,2\%, en segundo lugar ubican a los precios 21,7\%, luego la ambientación del sitio con 20,4\% y la ubicación con el 19,7\%. Estos resultados, resultan muy interesantes, para los servidores turísticos ya que orienta las acciones que se deben considerar para complementa las actividades turísticas principales y dar un servicio de calidad a los clientes.

Tabla 7 Aspectos que el cliente valora dentro del restaurante

\begin{tabular}{lcc}
\hline Opción & \multicolumn{2}{c}{ Frecuencia Porcentaje } \\
\hline Sabor & 54 & 26,7 \\
Presentación de platos & 37 & 18,3 \\
$\begin{array}{l}\text { Servicio y atención } \\
\text { cliente }\end{array}$ & al57 & 28,2 \\
Higiene y limpieza & 54 & 26,7 \\
Total & 202 & 100,0
\end{tabular}

Fuente: Encuesta a la demanda gastronómica

En la Tabla 7 se analizan los aspectos que los clientes valoran dentro de los restaurantes en primer lugar está el servicio y atención al cliente con el 28,2\%, seguido del sabor de los alimentos y la higiene y la limpieza con el 26,7\% para cada opción y en cuarto lugar se ubica la presentación de los platos. Esta valoración como la anterior son criterios generales que comúnmente el turista busca en el sitio en el que va a comer, sin embargo son argumentos importantes que debe ser considerados por los servidores de restauración para mejorar sus servicios.

Las variables que interfieren en la elección de un destino turístico por la gastronomía son diversas, en primer lugar esta un elemento culinario irrepetible e intransferible que ha sido a través del tiempo una representación de la vivencia del pueblo que le dio origen. Existen platos típicos que llegan a alcanzar esta denominación en un proceso de transiciones periféricas que no afectan su esencia, lo que les convierte en elementos representativos del patrimonio cultural.

La gastronomía de la ciudad de baños es el resultado de un encuentro de realidades diversas y en constate cambio, lo que obligan a cambios rápidos y radicales en las opciones culinarias con el propósito de cubrir las necesidades de miles de turistas que buscan en la adrenalina la satisfacción 
de su viaje y que asumen la gastronomía como un aspecto complementario que en el mejor de los casos le puede proporcionar una experiencia sensorial placentera.

\section{Discusión.}

Más allá de que la gastronomía sea un factor determinante para que los turistas elijan a la ciudad de Baños de Agua Santa, su desarrollo en la ciudad es importante por que se constituye en un elemento de soporte para las otras actividades turísticas y como tal contribuye con beneficios económicas importantes para los emprendedores que ofertan el servicio, al respecto Romo (2017), reporta que el 35,8\% de los restaurantes de la ciudad ofertan comida nacional y el 29,2\% comida internacional, al contrario de los resultados encontrados en esta investigación en donde la oferta de comida internacional supera a la oferta nacional en 12,5 puntos porcentuales, sin embargo se coincide en los precios de oferta de los platos en $\$ 4,00$ dolares promedio porque evidentemente son valores accesibles para el tipo de turistas que visitan la ciudad, obteniendo por este valor alimentos de muy buena calidad y un excelente servicio.

La exposición de los resultados de esta investigación hace referencia a la diversidad de oferta gastronómica de la ciudad de Baños de Agua Santa, por tal motivo se presentan algunos elementos que elevan los costos en preparación al respecto Romo (2017), en las conclusiones de su investigación considera "la compra de materia prima con el $67.5 \%$, debido que algunos productos son importados y por ende su precio es elevado, y $32.5 \%$ es la mano de obra e influyen en el beneficio económico" bajo estas consideraciones los gastos de los propietarios de los restaurantes se encuentran entre los \%6.000 y \$ 10.000 dólares al año.

El reporte de la investigación de Romo (2017), señala que luego de la aplicación de una análisis estadístico de Spearman (Rho) considerando las factores de tipo de gastronomía y los beneficios económicos como variables, la primera influye significativamente en la segundo, lo que significa que las beneficios económicos son importantes para los propietarios de los restaurantes. Este resultado es trascendente para justificar el desarrollo de la restauración en la ciudad de baños a pesar de no tener un plato gastronómicamente representativo, que motive a los turistas a elegir el destino por este motivo.

Ríos Ch (2015), en su investigación propone la difusión y posicionamiento de la melcocha como icono significativo en la gastronomía de la ciudad de Baños de Agua Santa, en su argumento asume que el posicionamiento de un producto gastronómico es fundamental para la promoción y difusión de un destino asegurando que se constituye en la motivación para que un turista elija un lugar de visita, en este aspecto, el análisis realizado en este trabajo ha demostrado múltiples ocasiones que esto es un hecho que sucede en muchos sitios, a nivel internacional e inclusive a nivel nacional, sin embargo detrás de este elemento se estructuran una serie de fatores operativos y valorativos que permiten que el solo hecho de ir a un sitio a degustar un producto sea motivo de viaje. Esta situación es especialísima, en la mayoría de destinos la gastronomía es un hecho complementario 
de otros atractivos y es desde no hace mucho tiempo que se le considera como un importante aporte de elemento sensoriales como factor determinante para la elección de un destino.

En este sentido la Melcocha o Alfeñique que se produce en la ciudad de Baños de Agua Santa, si bien es cierto, es un producto típico de la ciudad pero no exclusivo, carece de los elementos necesarios como para ser considerado como una especialidad gastronómica de suficiente valor como para que los turistas tomen la decisión de viajar al destino para degustarlo, se debe tomar en cuenta que el entorno que genera productos turísticos gastronómicos es un complejo constructo de elementos que favorecen o son complementarios, lo que sucede en la ciudad de Baños de Agua Santa con los deportes extremos.

Silva M (2016), realiza un trabajo interesante en la Identificación de nuevos productos gastronómicos en la ciudad de Baños de Agua Santa, establece a través de un estudio de las tradiciones culinarias de la ciudad de Baños de Agua Santa, los platos más representativos de la identidad del pueblo Baneño, los resultados que obtiene están basados en los datos proporcionados por restaurantes de gastronomía tradicional pero que están vinculados a la gastronomía de la Sierra Centro, se considera que la motivación de la autora de la investigación es loable y valedera, sin embargo, resulta complejo estructurar un entorno gastronómico que sea tan representativo al punto de convertirse en un factor determínate para que un turista elija un destino por esta razón, esto requiere de un proyecto de largo periodo en el que contribuyan todos los actores vinculados al, turismo y el producto gastronómico en ciernes se vaya consolidando paralelamente a otras actividades turísticas. Mientras tanto la actividad culinaria en la ciudad de Baños de Agua Santa debe ir evolucionando de tal manera que la propuesta gastronómica sea altamente significativa como experiencia sensorial, siguiendo los lineamientos que los principales destinos turísticos del mundo están adoptando.

\section{Conclusiones}

El turismo como actividad productiva a evolucionado en función de los múltiples y diversos requerimientos que los viajeros contemporáneos tienen. La globalización de la información, el desarrollo de los medios, la evolución del trasporte, son algunos de los factores que posibilitan que el turismo se diversifique de las más inimaginables formas y que sea posible hacerlo hasta en los lugares más recónditos del planeta.

La gastronomía, como elemento indisoluble del producto turístico esta asumiendo cada vez más un roll protagónico en la planificación de las actividades turísticas, ha dejado se ser el hecho necesario para satisfacer la necesidad de alimentarse del turista para convertirse en una experiencia sensorial significativa que busca dar al visitante placer y satisfacción de forma individual o integrándose a otros elementos de motivación turística.

Al formar parte de la estructura del producto turístico, la gastronomía es un factor importante en la consolidación de los productos y los destinos, constituyéndose en un elemento promocional 
insustituible y que va cobrando mayor interés por parte de los turistas y por lo tanto, está recibiendo un tratamiento especial por los organizadores y operadores de la actividad turística.

En la actualidad la gastronomía es tan importante dentro de 1 actividad turística que se están estructurando en el mundo turístico actividades alternativas para fortalecer esta área, con la implementación de festivales y rutas gastronómicas cuya aplicación implica mejorar la oferta turística en los destinos.

Se asume que la comida ocupa una buena parte de las actividades que el turista realiza durante su viaje, el hecho es que comer es una necesidad, sin embargo esta necesidad no necesariamente tiene que ser vacía y sin carga emocional, es el momento de compartir y a través de la degustación alcanzar un nivel de experiencia sensorial diferente y significativo.

Por sus características y vinculación con todo el encadenamiento de la actividad turística, los organizadores y operadores están utilizando a la gastronomía como parte de la promoción dándole al producto un valor agregado, encargado de motivar con mayor fuerza al visitante para que escoja su destino

El Ecuador es un país con una riqueza gastronómica incomparable, representada por platos típicos que se han convertido en iconos de las diferentes regiones, provincias y localidades, para los turistas locales, haciendo que sea una motivación adicional de viaje, pero por si misma no alcanza el nivel para ser el elemento principal que lo motive. En otras palabras, en el Ecuador, como en otros destinos turísticos del mundo, la gastronomía es un factor fundamental en la construcción del producto turístico, pero no es lo suficientemente importante como para constituirse en un factor determínate para la visita de turistas.

Esta situación es evidente en la Ciudad de Baños de Agua Santa, en donde la proliferación de actividades turísticas relacionadas sobre todo al turismo de aventura y a los deportes extremos a posibilitado un crecimiento y diversificación importante de la gastronomía, que sin tener un producto icono busca satisfacer las necesidades de los diferentes segmentos de turistas que llegan a la ciudad.

El análisis de la oferta y la demanda turística de la ciudad de Baños de agua Santa ha demostrado que la gastronomía no es factor determínate para la motivación de un turista al elegir este destino, más bien es un elemento complementario y necesario para el desarrollo de otras actividades turísticas preferidas por los visitantes

La gastronomía, es una actividad preexistente y prevalente que beneficia a un amplio sector de la población de la ciudad de Baños de Agua Santa, permitiéndoles tener una forma adecuada de vida. Es importante entonces mejorar y potenciar sus atributos con el propósito de contribuir equitativamente con los otros elementos que forman parte del destino turístico, para ello debe mantener la calidad de la comida y sostener la calidad en el servicio, de esta manera da el primer paso para ir descifrando y descubriendo una posible preparación gastronómica que se convierta en 
un motivo significativo para motivar a los turistas a visitar la ciudad de Baños de Agua Santa.

\section{Referencias bibliográficas.}

Arango R, N., Africano G, N., \& Viana A, R. (2018). La gastronomía colombiana. Atractivo turístico en crecimiento. Hitos de Ciencias Económico Administrativa, 68, 103-115.

Bailón, J., \& Rojas, R. (2017). Una Introducción al turismo gastronómico del Cantón MantaEcuador. Revista Internacional de Turismo, REmpresa y Territorio, 1(2), 25-32.

Curiel, E. (2015). Turismo gastronómico y enológico. MAdrid: Librertia Editorisl Dykinson.

Díaz M, M., \& García J, R. (2015). Turismo y tendencia. Oviedo: Septem Ediciones.

Enviajes. (2020). Los 10 Mejores Lugares Turísticos de Ecuador. Obtenido de Enviajes.cl: https://enviajes.cl/ecuador/lugares-turisticos-de-ecuador/

Falcón J, P. (2014). Tendencias globales de desarrollo del turismo gastronómico aplicadas al caso de argentina. Revista Acádemica de Marketig aplicado, 12, 35-67.

Franco C, M., Francio C, W., \& Torres N, M. (2017). Análisis de satisfacción. La gastronomía de Samborondón-Ecuador. Estudios Y perspectivas del Turismo, 26(2), 731-745.

Fusté F, F. (2017). La Gastronomía en el Marketing Turístico. Revista Anais Brasileiros de Estudos RTurísticos Abet, 88-99.

Hurtado V, M., \& Molina C, M. (2018). Análisis gastronómico de la ciudad de Baños de Agua Santa provincia de Tungurahua. Guayaquil: Universidad de Guayaquil.

Juárez L, O., \& Cebrián R, G. (2016). Estructura del Mercado Turístico 2. Madrid: Paraninfo.

Kido C, M., Carrión I, A., \& Kido C, A. (2018). La satisfacción del comensal como elemento clave del binomio gastronomía-turismo en Tijuana. Estudios sociales(Hermosillo Son), 28(51), Digital.

Kido C, M., Díaz C, I., \& Kido C, A. (2019). Una Nueva Interpretación de La satisfacción del comensal como elemento clave del binomio gastronomía-turismo en Tijuana. Estudios Sociales, 31(7), Digital.

Krauser R, W., \& Bahls A, D. (2016). A gastronomía como fator de influencia na escolha de destinações turísticas-base de estudo balneário Camboriú (SC-Brasil. Pasos- Revista de Turismoy Patrimonio Cultural, 14(2), 433-466.

López G, T., \& Jesus M, M. (2014). Turismo Cultura y Gastronomía. Una Aproximación a las rutas culinarias. Tourism y Management Studies, 1, 922-929. 
Masabanda T, E. (2015). La gastronomía y su incidencia en el desarrollo turístico-económico del cantón Baños de Agua Santa, provincia de Tungurahua. Ambato: UTA.

Mascarenhas R, G., \& Gândara J, M. (2015). O papel da gastronomía na qualidade e na competitividade dos destinos turísticos. CULTUR_Revista de Cultura e Turismo, 9(1), 6083.

Medina F, X. (2017). Reflexiones sobre el patrimonio y la alimentación desde las perspectivas cultural y turística. Anales de la Antropología, 51(2), 106-113.

MINTUR. (2018). Catastros Turisticos. Obtenido de Servicios Turismo: https://servicios.turismo.gob.ec/index.php/30-servicios-mintur/104-catastro-turistico

Norrid, A. (2017). Gastronomía y Turismo. Destinos con sal y pimienta. Rosa dos Ventos, 9(2), 308-314.

Povea T , N. (2018). Modelo de desarrollo turístico sostenible. Caso de estudio: Baños de Agua Santa. Quito: UTE.

Ricon , O., Santos, B., \& Galinmdo, L. (2016). Las mutaciones del comer: De McDonalds a los mundos ancestrales. Razón y Palabras, 20(94), 118-131.

Rios Ch, D. (2015). Difusión y posicionamiento de la melcocha como icono significativo en la gastronomía de la ciudad de Baños de Agua Santa en la provincia de Tungurahua en el año 2013. Ambato: UTA.

Rivera P, M. (2013). Elaboración de la guía gastronómica de la cocina tradicional del cantón Baños de Agua Santa, provincia de Tungurahua. Ambato: UNIANDES.

Romo M, M. (2017). ). Medición del beneficio económico generado en la gastronomía del destino turístico Baños de Agua Santa. Ambato: UTA.

Serra J, R., \& Pascual M, M. (s/f). Patrimonio alimentario, turismo y espectáculo. reflexiones en torno a un proyecto de desarrollo de experiencias turísticas gastronómicas. Barcelona: EDiciones de la Universidad de Barcelona.

Silva S, M. (2016). Identificación de nuevos productos gastronómicos en la ciudad de Baños de Agua Santa. Ambato: UNIANDES.

Sobrado D, A. (2018). dentidad cultural y turismo gastronómico: la mercantilización del patrimonio. International journal of scientific management and tourism, 4(2), 51-71.

Uribe A, C., Avalos E, A., \& Villa J, M. (2017). Educación en gastronomía: su vínculo con la identidad cultural y el turismo. El periplo Sustentable: Revista de Turismo, Desarrollo y Competitividad, 32, 1-2. 
Varisco , C. (2015). La Cadena productiva del Turismo. Portal de Promoción y Difusión pública del Conocimiento Académico y Científico, 59-85.

Vázquez M, U. (2017). Place branding. La gastronomía como valor de marca y factor de atracción turística: el caso de España. Madrid: ESIC Editorial. 
PARA CITAR EL ARTÍCULO INDEXADO.

Robalino Vallejo, J. A., Rivadeneira Yánez, C. F., Moreno Guerra, A. M., \& Fierro Ricaurte, A. E. (2020). Incidencia de la gastronomía para elegir un destino. Caso de estudio: Baños de Agua Santa. Tungurahua-Ecuador. $\quad$ ConcienciaDigital, $\quad 3(2.1), \quad 6-24$. https://doi.org/10.33262/concienciadigital.v3i2.1.1217

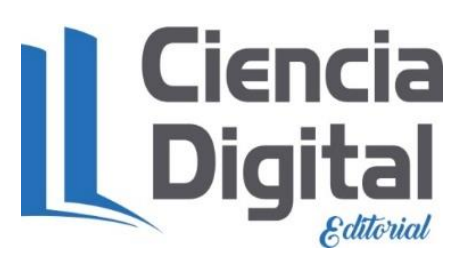

El artículo que se publica es de exclusiva responsabilidad de los autores y no necesariamente reflejan el pensamiento de la Revista Conciencia Digital.

El artículo queda en propiedad de la revista y, por tanto, su publicación parcial y/o total en otro medio tiene que ser autorizado por el director de la Revista Conciencia Digital.
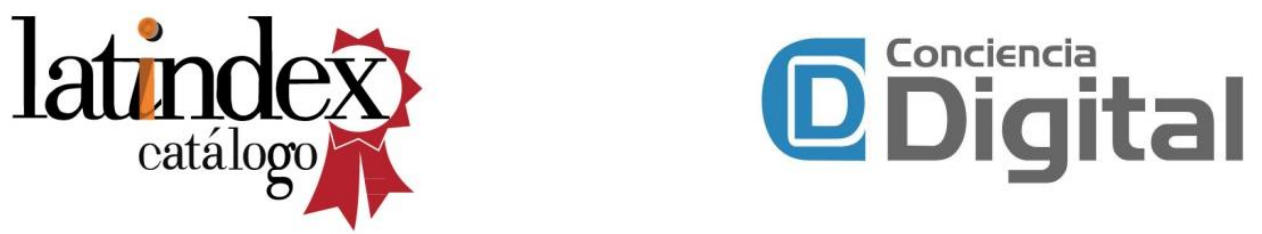